\title{
A genetic algorithm for the partial binary constraint satisfaction problem: an application to a frequency assignment problem
}

Citation for published version (APA):

Kolen, A. W. J. (2006). A genetic algorithm for the partial binary constraint satisfaction problem: an application to a frequency assignment problem. METEOR, Maastricht University School of Business and Economics. METEOR Research Memorandum No. 045 https://doi.org/10.26481/umamet.2006045

Document status and date:

Published: 01/01/2006

DOI:

10.26481/umamet.2006045

Document Version:

Publisher's PDF, also known as Version of record

Please check the document version of this publication:

- A submitted manuscript is the version of the article upon submission and before peer-review. There can be important differences between the submitted version and the official published version of record.

People interested in the research are advised to contact the author for the final version of the publication, or visit the DOI to the publisher's website.

- The final author version and the galley proof are versions of the publication after peer review.

- The final published version features the final layout of the paper including the volume, issue and page numbers.

Link to publication

\footnotetext{
General rights rights.

- You may freely distribute the URL identifying the publication in the public portal. please follow below link for the End User Agreement:

www.umlib.nl/taverne-license

Take down policy

If you believe that this document breaches copyright please contact us at:

repository@maastrichtuniversity.nl

providing details and we will investigate your claim.
}

Copyright and moral rights for the publications made accessible in the public portal are retained by the authors and/or other copyright owners and it is a condition of accessing publications that users recognise and abide by the legal requirements associated with these

- Users may download and print one copy of any publication from the public portal for the purpose of private study or research.

- You may not further distribute the material or use it for any profit-making activity or commercial gain

If the publication is distributed under the terms of Article $25 \mathrm{fa}$ of the Dutch Copyright Act, indicated by the "Taverne" license above, 
Antoon Kolen

A genetic algorithm for the partial binary constraint satisfaction problem: an application to a frequency assignment problem

$\mathrm{RM} / 06 / 045$

JEL code: C61

\section{METEबrR}

Maastricht research school of Economics of TEchnology and ORganizations

Universiteit Maastricht

Faculty of Economics and Business Administration

P.O. Box 616

NL - 6200 MD Maastricht

phone : ++31 433883830

fax : $\quad++31433884873$ 



\title{
A genetic algorithm for the partial binary constraint satisfaction problem: an application to a frequency assignment problem.
}

\author{
Antoon Kolen *
}

October 12, 2006

\begin{abstract}
We describe a genetic algorithm for the partial constraint satisfaction problem. The typical elements of a genetic algorithm, selection, mutation and cross-over, are filled in with combinatorial ideas. For instance, cross-over of two solutions is performed by taking the one or two domain elements in the solutions of each of the variables as the complete domain of the variable. Then a branch-and-bound method is used for solving this small instance. When tested on a class of frequency assignment problems this genetic algorithm produced the best known solutions for all test problems. This feeds the idea that combinatorial ideas may well be useful in genetic algorithms.
\end{abstract}

\section{Introduction}

A constraint satisfaction problem (CSP) is a problem defined by a finite set of variables, each of which has a finite set of possible values (the domain). Next, a set of constraints defined on these variables is given, which determine feasible combinations of domain elements of multiple variables. We consider only binary constraints, where the number of variables involved in each constraint is restricted to two. So, a constraint may forbid only pairs of domain elements. A solution of a binary constraint satisfaction problem consists of exactly one domain value in the domain of each variable, such that no forbidden combinations are present. We add one more feature to the binary constraint satisfaction problem (BCSP), namely penalties for domain values in the domain elements and penalties for pairs of domain elements. The value of a solution is the sum of the penalties. The objective of this partial binary constraint satisfaction problem (PBCSP) is to find a solution of minimum value. Note that standard constraints can be modeled with penalties by incurring very high penalties for forbidden combinations. Therefore, in the sequel we will only consider constraints with penalties.

In [3] genetic algorithms have been described for CSP problems. We develop a genetic algorithm specifically suitable for PBCSP. The main feature of this algorithm is the procedure for cross-over. Here two solutions, the parents, are selected from a population. The one or two values of each variable in these solutions are taken as the domain of the variable. Then, an exact algorithm will solve this small PBCSP to optimality. Note that

\footnotetext{
*Antoon Kolen deceased on October 3, 2004. This manuscript has been updated by Stan van Hoesel, Faculty of Economics and Business Administration, University Maastricht, P.O. Box 616, 6200 MD Maastricht, The Netherlands, Email: s.vanhoesel@ke.unimaas.nl
} 
this means that the offspring solution is at least as good as each of the parents. Thus, if each of the solutions in the parent population is selected at least once, the offspring population is at least as good as the parent population. The genetic algorithm is tested on a set of problems, arising in frequency assignment.

In section 2 we will formally describe the PBCSP, and give examples. In Section 3 we describe the genetic algorithm for the partial constraint satisfaction problem, where as in Section 4 we solve the cross-over problem, and in Section 5 we apply the genetic algorithm to the RLFAP and discuss computational results for the CALMA -instances.

\section{The PBCSP and examples: Frequency Assignment and MAX-SAT}

Formally, a PBCSP can be described with the following tuple: $\left(G=(V, E), D_{V}, Q_{V}, P_{E}\right)$. Here,

1. $G=(V, E)$, an undirected graph called the constraint graph, the vertices correspond to the variables, and the edges correspond to the constraints.

2. $D_{V}=\left\{D_{v} \mid v \in V\right\}$, where $D_{v}$ is a finite set, called the domain of variable $v \in V$,

3. $Q_{V}=\left\{Q_{v}: D_{v} \mapsto \mathbb{R} \mid v \in V\right\}$, where $Q_{v}$ defines a variable penalty function which assigns a real value to every domain value in $D_{v}, v \in V$,

4. $P_{E}=\left\{P_{\{v, w\}}: D_{v} \times D_{w} \mapsto \mathbb{R} \mid\{v, w\} \in E\right\}$, where $P_{\{v, w\}}$ defines a constraint penalty function which assigns a real value to every pair of domain elements in $D_{v} \times D_{w}$, $\{v, w\} \in E$.

A solution is defined to be a vector $\left(d_{v}\right)_{v \in V}$ containing exactly one domain element $d_{v} \in$ $D_{v}$ for every vertex $v \in V$. The value of a solution $\left(d_{v}\right)_{v \in V}$ is defined to be the sum of all vertex and edge penalties, i.e., $\sum_{v \in V} Q_{v}\left(d_{v}\right)+\sum_{\{v, w\} \in E} P_{\{v, w\}}\left(\left\{d_{v}, d_{w}\right\}\right)$. The objective is to find a solution of minimum value.

The PBCSP can be formulated as a $\{0,1\}$-programming problem. Let us define for all $v \in V$ and all $d_{v} \in D_{v}$ the $\{0,1\}$-variables

$$
y\left(v, d_{v}\right)= \begin{cases}1 & \text { if } d_{v} \in D_{v} \text { is selected } \\ 0 & \text { otherwise }\end{cases}
$$

and for all $\{v, w\} \in E, d_{v} \in D_{v}$ and $d_{w} \in D_{w}$ the $\{0,1\}$-variables

$$
x\left(v, d_{v}, w, d_{w}\right)= \begin{cases}1 & \text { if }\left\{d_{v}, d_{w}\right\} \in D_{v} \times D_{w} \text { is selected } \\ 0 & \text { otherwise }\end{cases}
$$

Furthermore, let us define for all $v \in V$ and for all $d_{v} \in D_{v} q\left(v, d_{v}\right)=Q_{v}\left(d_{v}\right)$ and for all $\{v, w\} \in E$, for all $d_{v} \in D_{v}$ and for all $d_{w} \in D_{w} p\left(v, d_{v}, w, d_{w}\right)=P_{\{v, w\}}\left(\left\{d_{v}, d_{w}\right\}\right)$.

Then PBCSP can be formulated as a 


$$
\begin{aligned}
& \min : \sum_{v \in V} \sum_{d_{v} \in D_{v}} q\left(v, d_{v}\right) y\left(v, d_{v}\right)+ \\
& \sum_{\{v, w\} \in E} \sum_{d_{v} \in D_{v}} \sum_{d_{w} \in D_{w}} p\left(v, d_{v}, w, d_{w}\right) x\left(v, d_{v}, w, d_{w}\right)
\end{aligned}
$$

s.t.

$$
\begin{array}{ll}
\sum_{d_{v} \in D_{v}} y\left(v, d_{v}\right)=1 & \forall_{v \in V} \\
\sum_{d_{w} \in D_{w}} x\left(v, d_{v}, w, d_{w}\right)=y\left(v, d_{v}\right) & \forall_{\{v, w\} \in E} \forall_{d_{v} \in D_{v}} \\
y\left(v, d_{v}\right) \in\{0,1\} & \forall_{v \in V} \forall_{d_{v} \in D_{v}} \\
x\left(v, d_{v}, w, d_{w}\right) \in\{0,1\} & \forall_{\{v, w\} \in E} \forall_{d_{v} \in D_{v}} \forall_{d_{w} \in D_{w}}
\end{array}
$$

The first set of constraints expresses that exactly one value in the domain of every variable must be selected. The next set of constraints expresses the following: if $d_{v}$ is selected then for exactly one combination $d_{v} d_{w} \in D_{w}$ is selected. If $d_{v}$ is not selected, then also none of the combinations $d_{v} d_{w} \in D_{w}$ is selected.

\section{$2.1 \quad$ Frequency assignment}

A straightforward example of a PBCSP is the radio link frequency assignment problem studied in the CALMA-project. In the CALMA (Combinatorial ALgorithms for Military Applications)-project researchers from England, France, and the Netherlands tested different combinatorial algorithms on a set of 11 frequency assignment problems. The genetic algorithm to be described in this paper found the best known solutions to all test problems and for some test problems outperforms other solution techniques. Results of the CALMAproject as well as all test problems are described in [2] and available by anonymous ftp from ftp.win.tue.nl in the directory /pub/techreports/CALMA.

The radio link frequency assignment problem (RLFAP) is defined by a set of radio links partitioned into pairs, where each pair consists of a receiver and a transmitter radio link. Associated with a radio link is a set of frequencies which can be assigned to it. The distance between the frequencies assigned to the receiver and transmitter must be equal to a fixed distance. For some pairs of radio links there is an interference constraint which states that the distance between the two assigned frequencies should be greater than a given required distance. It is a soft constraint because it may be violated at a certain interference cost. Both the required distance and the associated cost depend on the two radio links involved. For some receiver-transmitter pairs a preferred pair of frequencies is given. It is possible to deviate from these preferred frequencies at a certain mobility cost which depends on the receiver-transmitter pair. The objective is to find a frequency assignment that minimizes total cost, i.e., the sum of total interference cost and total mobility cost. An overview on models and algorithms for frequency assignment can be found in [1].

To model RLFAP as a PBCSP we introduce a variable for every receiver-transmitter pair. The domain of a variable consists of every pair of frequencies, one from the domain of the receiver radio link and one from the transmitter radio link, which satisfy the fixed distance requirement. The variable penalty of a pair of frequencies is equal to the mobility cost. There is a constraint for two receiver-transmitter pairs when there is at least one interference constraint between two radio links one from each receiver-transmitter pair. 
The constraint penalty for a pair is equal to the sum of the interference cost of the four involved radio links (two receivers-transmitter pairs). For two receiver-transmitter pairs the mutual distance requirements and interference cost are given below.

\begin{tabular}{|r|r|r|}
\hline & $\mathrm{r}_{2}$ & $\mathrm{t}_{2}$ \\
\hline $\mathrm{r}_{1}$ & 351 & 518 \\
\hline $\mathrm{t}_{1}$ & 201 & 351 \\
\hline \multicolumn{3}{|c}{ distances }
\end{tabular}

\begin{tabular}{|r|r|r|}
\hline & $\mathrm{r}_{2}$ & $\mathrm{t}_{2}$ \\
\hline $\mathrm{r}_{1}$ & 1000 & 10 \\
\hline $\mathrm{t}_{1}$ & 1 & 1000 \\
\hline \multicolumn{3}{|c}{ costs }
\end{tabular}

For the CALMA instance the distance between the frequencies assigned to a receiver pair must be equal to 238 . If we assign $(72,310)$ to $\left(\mathrm{r}_{1}, \mathrm{t}_{1}\right)$ and $(414,652)$ to $\left(\mathrm{r}_{2}, \mathrm{t}_{2}\right)$, then according to the tables above the corresponding constraint penalty is 1000 (for $r_{1}$ and $r_{2}$ ) $+1\left(\right.$ for $t_{1}$ and $\left.r_{2}\right)=1001$.

\subsection{MAX-SAT}

The maximum satisfiability problem (MAX-SAT) can be modeled as a PCSP. Let $C_{1}, C_{2}, \ldots, C_{m}$ be a set of clauses defined on the boolean variables $x_{1}, x_{2}, . ., x_{n}$. Each clause is a disjunction of literals, where a literal is either a boolean variable $x_{i}$ or its negation $\bar{x}_{i}$. The objective is to assign to each boolean variable the value true or false so as to satisfy the maximum number of clauses or equivalently minimize the number of clauses that are not satisfied. A clause is satisfied if and only if at least one literal in the clause has the value true. An example of the maximum satisfiability problem is given by

\section{Example 1.}

$$
\left(x_{1} \vee x_{3}\right) \wedge\left(x_{1} \vee x_{2} \vee x_{3}\right) \wedge\left(x_{2} \vee \bar{x}_{3}\right)
$$

$$
C_{1} \quad C_{2} \quad C_{3}
$$

To model the maximum satisfiability problem as a PBCSP we introduce a variable for each clause and a variable for each boolean variable. The domain of a clause variable contains the literals in the clause. We denote a domain element by the index of corresponding literal. The domain of a variable corresponding to a boolean variable consists of the values true and false. There is a constraint between a variable corresponding to a clause and a variable corresponding to a boolean variable whenever the boolean variable or its negation occurs in the clause.

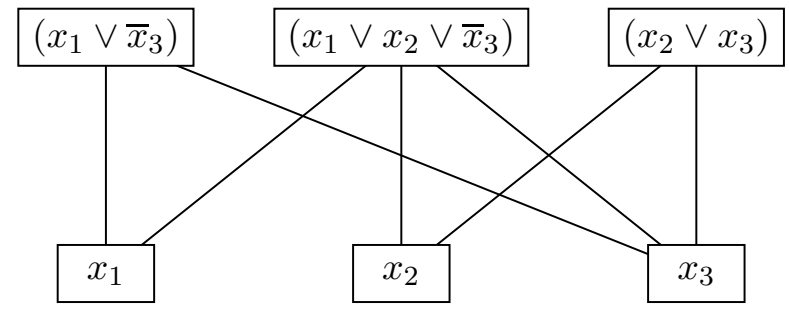

Figure 1: Constraints in example MAX-SAT instance.

The constraint penalty of any pair of conflicting domain values is equal to one; all other constraint penalties are zero. So, the domain pair $\left\{x_{i}\right.$, false $\}$ is conflicting, and thus incurs a penalty of one. The same holds for the pair $\left\{\bar{x}_{i}\right.$, true $\}$. There are no other penalties. Figure 2 shows the variables and constraints corresponding to Example 1 where conflicting pairs of domain values are indicated by a line. 


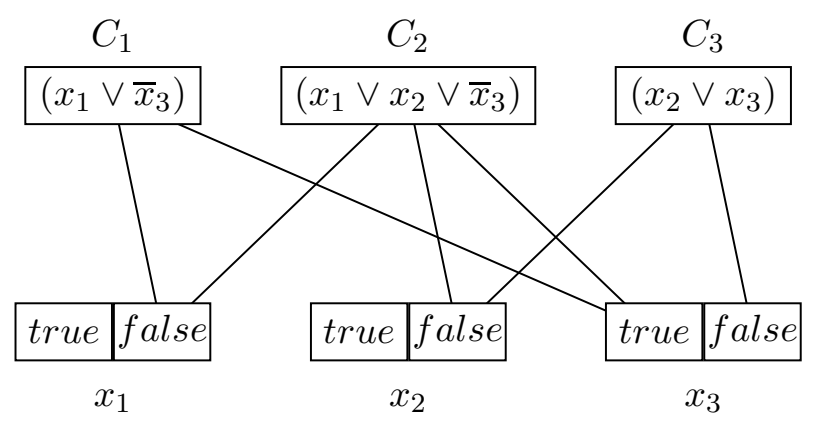

Figure 2: Penalties in example MAX-SAT instance.

Theorem 1. Consider an instance of MAX-SAT with $n$ variables and $m$ clauses. Then, $M A X-S A T$ has value $k$ if and only if the corresponding instance of PBCSP has value $m-k$.

Proof. Consider a truth assignment of the boolean variables of the maximum satisfiability problem that satisfies $k$ of the $m$ clauses. In the corresponding partial binary constraint satisfaction problem we select the domain values of the variable corresponding to boolean variables as defined by the truth assignment. For the $k$ clauses that are satisfied we select a literal that has the value true. For the $m-k$ clauses that are not satisfied we select an arbitrary literal. Then the value of this solution is $m-k$. This proves that, if the optimal value of the maximum satisfiability problem is $k$, then the optimal value of the partial binary constraint satisfaction problem is at most $m-k$.

Consider an optimal solution of the partial constraint problem with value $m-k$. Then there are exactly $m-k$. clauses for which the selected literal is involved in a conflict and since the solution is optimal any other literal selected in the same clause would result in a conflict as well. Hence the domain values of the variables corresponding to boolean variables define a truth assignment that satisfies exactly $k$ clauses. We conclude that if the optimal value of the partial binary constraint problem is $m-k$, then the optimal value of the maximum satisfiability problem is at least $k$.

If for Example 1 we consider the truth assignment $x_{1}=$ true, $x_{2}=$ false, $x_{3}=$ false, then $C_{1}$ and $C_{2}$ are satisfied, and $C_{3}$ is violated. For the partial binary constraint satisfaction problem we select true for $x_{1}$, false for $x_{2}$, false for $x_{3}, x_{1}$ for $C_{1}, x_{1}$ for $C_{2}$, and $x_{2}$ for $C_{3}$. If in Figure 1 we select $x_{1}$ for $C_{1}, x_{1}$ for $C_{2}, x_{2}$ for $C_{3}$, true for $x_{1}$,true for $x_{2}$, and false for $x_{3}$, then we have the optimal solution of the partial binary constraint satisfaction problem of value zero. The corresponding truth assignment $x_{1}=$ true, $x_{2}=$ true, and $x_{3}=$ false satisfies all clauses.

We conclude from the above results that the optimal value of the partial binary constraint satisfaction problem is $m-k$ if and only if the optimal value of the maximum satisfiability problem is $k$.

\section{The genetic algorithm.}

Local search algorithms, such as simulated annealing and tabu search, construct a sequence of solutions, where the successor of a given solution in the sequence is a so-called neighbor of that solution. The definition of the neighborhood of a given solution is a basic ingredient of any local search algorithm. 
A genetic algorithm constructs a sequence of populations of solutions, where the successor of a given population in the sequence is a so-called neighbor of that population. A population consists of a set of solutions. The basic ingredients of a genetic algorithm are selection, cross-over, and mutation procedures. A population is called a neighbor of a given population if it can be obtained from that population by applying these procedures to the given population. A new population is constructed from an old population by repeatedly applying cross-over to pairs of elements of the old population selected by the selection procedure. The idea of cross-over is to use the genetic material of the two input elements to construct two elements of the new population, which are hopefully better than the two input elements. The mutation procedure changes a given solution. For a more detailed discussion of genetic algorithms we refer to the book by Goldberg (1988). No previous knowledge of genetic algorithms is required to understand the description of our genetic algorithm.

Let us call two solutions neighbors if they differ in exactly one component, i.e., one domain element. A 1-optimal solution is a solution with the property that no neighbor exists with a smaller value. An element of our genetic algorithm is defined to be a 1-optimal solution. A population of our genetic algorithm consists of a fixed number (called the population size) of (not necessarily distinct) elements. The initial population of our genetic algorithm is constructed by generating random solutions, and by applying a local optimization algorithm to each solution to make it 1-optimal. Our genetic algorithm consists of a number of generations. Each generation starts with a given population, called the parent population, and constructs a new population of the same size, the offspring. This offspring population is the parent population of the next generation. The offspring population will have the property that it is at least as good as the parent population in the sense that every element of the parent population is replaced by an element with a value which is less than or equal to the value of the element it replaces. The genetic algorithm returns the element of minimum value in the last population.

To construct a parent population from the offspring population we randomly select for each element of the old population a partner element from the offspring population. This partner element is selected with a probability which is proportional to the reciprocal of its value. This means that elements with a small value are preferred in the selection procedure. Next we apply the cross-over function to the given element and the random selected partner element. The cross-over function takes as input two elements (called parents) and returns an element (called offspring) with a value that is less than or equal to the value of both input elements. So, in this cross-over only one offspring is created, in contrast with the two offspring in traditional cross-over. After applying a local optimization procedure (the mutation procedure) to make this offspring 1-optimal it will replace the given element in the parent population. The construction of the parent population from the offspring population is summarized below.

for $\mathrm{i}:=1$ to PopSize do

\{

(Selection)

Select ParentPopulation[i] and random select ParentPopulation[j] with a probability that is inverse proportional to its objective value.

(Cross-over)

OffspringPopulation[i] := CrossOver(ParentPopulation[i],ParentPopulation[j]); 
(Mutation)

Local optimize OffspringPopulation[i] to make it 1-optimal;

\}

The idea of the cross-over is that each domain value of the offspring will be inherited from one of its parents. Given two parent solutions we can consider the restricted PBCSP where the domain of a variable contains the set of domain values assigned to it in one of the two parents. So the domain of a variable contains exactly one domain value if it is assigned the same domain value in both parents, otherwise the domain consists of the two different domain values assigned to it in both parents. The offspring of our cross-over procedure will be the optimal solution of this restricted partial constraint satisfaction problem. Since both parents are solutions of this problem, the optimal solution, i.e., the offspring, will have a value which is less than or equal to the minimum value of its parents. We will discuss an exact solution method of the restricted PBCSP in Section 4.

Our genetic algorithm as described above differs from more traditional genetic algorithms in two aspects. In a traditional genetic algorithm a cross-over produces two offsprings instead of the one offspring as in our cross-over. In a traditional genetic algorithm both parents to which the cross-over is applied are selected randomly. The probability of being selected is determined by a so-called fitness function. In our genetic algorithm the fitness of an element is defined as the reciprocal of its value. Although we have experimented with more traditional genetic algorithms using our cross-over the best results were obtained using the version as described above. An intuitive explanation for the success of our algorithm may be that every element of the population is involved in a cross-over at least once, while good solutions have a high probability of being involved in more than one cross-over. So although we prefer good genetic material (i.e., domain values) we do not exclude any genetic material beforehand. The local optimization procedure we apply to the offspring of the cross-over procedure to make it 1-optimal is used as the mutation operator of our genetic algorithm.

\section{The cross-over}

Consider the $\{0,1\}$-programming formulation of PBCSP of section 2. Before solving this problem we first apply some preprocessing techniques to reduce the size of the problem. During the preprocessing we will always make sure that for every edge $\{v, w\} \in E$ with $D_{v}=\left\{a_{v}, b_{v}\right\}$ and $D_{w}=\left\{a_{w}, b_{w}\right\}$ the edge penalties satisfy $\min \left\{p\left(v, a_{v}, w, a_{w}\right), p\left(v, a_{v}, w, b_{w}\right)\right\}=$ $0, \min \left\{p\left(v, b_{v}, w, a_{w}\right), p\left(v, b_{v}, w, b_{w}\right)\right\}=0, \min \left\{p\left(v, a_{v}, w, a_{w}\right), p\left(v, b_{v}, w, a_{w}\right)\right\}=0$, and $\min \left\{p\left(v, a_{v}, w, b_{w}\right), p\left(v, b_{v}, w, b_{w}\right)\right\}=0$.

Assume that these conditions do not hold, say $\min \left\{p\left(v, a_{v}, w, a_{w}\right), p\left(v, a_{v}, w, b_{w}\right)\right\}>0$. Then we can increase the vertex penalty $q\left(v, a_{v}\right)$ with $\min \left\{p\left(v, a_{v}, w, a_{w}\right), p\left(v, a_{v}, w, b_{w}\right)\right\}$ and decrease both $p\left(v, a_{v}, w, a_{w}\right)$ and $p\left(v, a_{v}, w, b_{w}\right)$ with $\min \left\{p\left(v, a_{v}, w, a_{w}\right), p\left(v, a_{v}, w, b_{w}\right)\right\}$ without affecting the total penalty of a solution.

We use five different types of preprocessing techniques, namely deleting constraints, deleting variables with a domain of cardinality one, deleting variables of degree one in the constraint graph, deleting variables of degree two in the constraint graph, and deleting dominated domain values. Although we will discuss these preprocessing techniques in the context of our cross-over problem, they apply to any partial binary constraint satisfaction problem.

1. A constraint is deleted whenever all constraint penalties are equal 
2. Consider a variable $v \in V$ with a domain of cardinality one, say $D_{v}=\left\{a_{v}\right\}$.

For an edge $\{v, w\} \in E$ the edge penalty only depends on which $d_{w}$ is selected from $D_{w}$. Therefore the edge penalty $p\left(v, a_{v}, w, d_{w}\right)$ can be viewed as a vertex penalty for $d_{w}$. If we define $q\left(w, d_{w}\right):=q\left(w, d_{w}\right)+p\left(v, a_{v}, w, d_{w}\right)$ for all $\{v, w\} \in E$ and for all $d_{w} \in D_{w}$, and delete vertex $v$ and all edges incident to it from the constraint graph, then there is a one-to-one correspondence between solutions of the reduced problem and solutions of the original problem. The objective value of a solution to the original problem is equal to the objective value of the corresponding problem of the reduced problem plus $q\left(v, a_{v}\right)$.

3. Consider a variable $v \in V$ of degree one in the constraint graph, and let $\{v, w\}$ the edge incident to $v$.

If $d_{w} \in D_{w}$ is assigned to $w$, then the domain value $d_{v}^{*} \in D_{v}$ assigned to $v$ will be selected so as to minimize the sum of the vertex penalty of $v$ and the edge penalty of $\{v, w\}$, i.e., $q\left(v, d_{v}^{*}\right)+p\left(v, d_{v}^{*}, w, d_{w}\right)=\min \left\{q\left(v, d_{v}\right)+p\left(v, d_{v}, w, d_{w}\right) \mid d_{v} \in D_{v}\right\}$. If we define $q\left(w, d_{w}\right):=q\left(w, d_{w}\right)+\min \left\{q\left(v, d_{v}\right)+p\left(v, d_{v}, w, d_{w}\right) \mid d_{v} \in D_{v}\right\}$, and delete vertex $v$ and the edge $\{v, w\}$, then the optimal value of the reduced and original problem are the same. Given an optimal solution of the reduced problem with $d_{w}$ assigned to $w$ we can find an optimal solution to the original problem by assigning the domain value $d_{v}^{*}$ defined above to $v$.

4. Consider a variable $v \in V$ of degree two in the constraint graph, and let $\{u, v\}$ and $\{v, w\}$ the edges incident to $v$.

If $d_{u} \in D_{u}$ is assigned to $u$ and $d_{w} \in D_{w}$ is assigned to $w$, then the domain value $d_{v}^{*} \in D_{v}$ assigned to $v$ will be selected so as to minimize the sum of the vertex penalty of $v$ and the edge penalties of $\{u, v\}$ and $\{v, w\}$, i.e, $p\left(u, d_{u}, v, d_{v}^{*}\right)+q\left(v, d_{v}^{*}\right)+$ $p\left(v, d_{v}^{*}, w, d_{w}\right)=\min \left\{p\left(u, d_{u}, v, d_{v}\right)+q\left(v, d_{v}\right)+p\left(v, d_{v}, w, d_{w}\right) \mid d_{v} \in D_{v}\right\}$. If there is no edge $\{u, w\}$ then we add the edge $\{u, w\}$ with all edge penalties equal to zero to the constraint graph. Clearly this does not affect the value of any solution. If we now delete the edges $\{u, v\}$ and $\{v, w\}$ from the constraint graph and define $p\left(u, d_{u}, w, d_{w}\right):=p\left(u, d_{u}, w, d_{w}\right)+\min \left\{p\left(u, d_{u}, v, d_{v}\right)+q\left(v, d_{v}\right)+p\left(v, d_{v}, w, d_{w}\right) \mid d_{v} \in\right.$ $\left.D_{v}\right\}$, then the optimal value of the reduced and original problem are the same. Given an optimal solution of the reduced problem with $d_{u}$ assigned to $u$ and $d_{w}$ assigned to $w$ we can find an optimal solution to the original problem by assigning the domain value $d_{v}^{*}$ defined above to $v$.

5. Our last preprocessing technique deals with dominated domain values.

For a variable $v \in V$ we say that domain value $b_{v} \in D_{v}$ dominates domain value $a_{v} \in D_{v}$ whenever for every solution in which $a_{v}$ is assigned to $v$ we can find a solution in which $b_{v}$ is assigned to $v$, and for which the latter solution has a value that is less than or equal to the value of the former solution.

If $b_{v}$ dominates $a_{v}$, then $a_{v}$ can be deleted from the domain $D_{v}$ of vertex $v$. If the original domain $D_{v}$ has cardinality two, then the reduced domain has cardinality one and vertex $v$ can be deleted using preprocessing technique 2 .

We will formulate a sufficient condition for dominance.

If $\{v, w\}$ is an edge, then the worst increase in the edge penalty for this edge if $a_{v}$ in a solution is replaced by $b_{v}$ is given by $c(v, w)=\max \left\{p\left(v, b_{v}, w, d_{w}\right)-p\left(v, a_{v}, w, d_{w}\right) \mid d_{w} \in\right.$ 
$\left.D_{w}\right\}$. If $q\left(v, b_{v}\right)+\sum_{\{v, w\} \in E} c(v, w) \leq q\left(v, a_{v}\right)$, then $b_{v}$ dominates $a_{v}$ because replacing $a_{v}$ by $b_{v}$ in any solution will result in a solution which is at least as good. For any variable with a domain of cardinality two we check whether one domain value is dominated by the other domain value by checking the sufficient condition described above. If a domain value is dominated, then it is deleted from the domain.

After application of all preprocessing techniques the $\{0,1\}$-programming problem of the remaining partial binary constraint satisfaction problem is solved by a partial cutting plane algorithm. The class of valid inequalities that we use in the cutting plane algorithm is one of the classes defined in [4]. The inequalities are called 3-cycle inequalities.

Given vertices $u, v$, and $w$ which form a 3 -cycle in the constraint graph. Let the domains be given by $D_{u}=\left\{a_{u}, b_{u}\right\}, D_{v}=\left\{a_{v}, b_{v}\right\}$, and $D_{w}=\left\{a_{w}, b_{w}\right\}$. Then it is easy to see that the following 3 -cycle inequality is a valid inequality

$$
x\left(u, a_{u}, v, b_{v}\right)+x\left(v, a_{v}, w, b_{w}\right)+x\left(w, a_{w}, u, b_{u}\right)<=1 .
$$

For a given 3-cycle there are four 3-cycle inequalities of this type which are facet defining for the convex hull of solutions of the partial binary satisfaction problem.. We start the solution procedure by first solving the linear programming relaxation. Then we add all 3 -cycle inequalities that are violated by the current optimal linear programming solutions and re-optimize. This is repeated until all 3-cycle inequalities are satisfied. In almost all problems we solved we end with an integer optimal solution at this stage. If the optimal linear programming solution is fractional, then we solve the original $\{0,1\}$-programming problem with all added 3-cycle inequalities using CPlex 4.0 as mixed integer optimizer.

\section{Computational results.}

In the CALMA project two sets of problem instances were used. The CELAR instances are provided by the French ministry of defense. The GRAPH instances are random instances, generated by a group of researchers from Delft University of Technology, which have the same characteristics as the CELAR instances.

There were four problem instances (CELAR 9, CELAR 10, GRAPH 7, and GRAPH 12) which involved mobility penalties. These problem instances turned out to be very easy, and all tested solution techniques found the same answers (CELAR 9: 15571, CELAR 10: 31516, GRAPH 7: 4324, GRAPH 12: 11827). The genetic algorithm already found these solutions within 5 generations with a population size of 10 . Therefore these problem instances are left out of the computational tests.

In Table 1 we list for each problem instance of our problem type its name as referred to in the CALMA project, the number of vertices $(|V|)$ and the number of edges $(|E|)$, the number of y-variables, which can be calculated from $\sum_{v \in V}\left|D_{v}\right| /|V|$, the number of X-variables, which can be calculated from $\sum_{\{v, w\} \in e}\left|D_{v}\right| *\left|D_{w}\right| /|E|$, and the best known solution value $(O)$ for each problem. The best known solutions are obtained by the genetic algorithm described in the previous section.

Our genetic algorithm was implemented in $\mathrm{C}++$ and run on a workstation (DEC 2100 A500MP). All linear and mixed integer program are solved using CPLEX 4.0. Although the computational time of our genetic algorithm already is very impressive, the code for the cross-over function is not yet fully optimized. Since the cross-over function is called many times, there is still room for improvement. 


\begin{tabular}{|r|r|r|r|r|r|}
\hline Name & $|V|$ & $|E|$ & $\sum_{v \in V}\left|D_{v}\right| /|V|$ & $\sum_{\{v, w\} \in E}\left(\left|D_{v}\right|\left|D_{w}\right|\right) /|E|$ & \multicolumn{1}{l|}{$\mathrm{O}$} \\
\hline CELAR 6 & 100 & 350 & 40.10 & 1622.58 & 3389 \\
\hline CELAR 7 & 200 & 817 & 39.88 & 1619.43 & 343592 \\
\hline CELAR 8 & 458 & 1655 & 39.52 & 1510.91 & 262 \\
\hline GRAPH 5 & 100 & 416 & 37.08 & 1372.40 & 221 \\
\hline GRAPH 6 & 200 & 843 & 37.71 & 1416.48 & 4115 \\
\hline GRAPH 11 & 340 & 1425 & 37.70 & 1417.52 & 3080 \\
\hline GRAPH 13 & 458 & 1877 & 38.40 & 1481.52 & 10110 \\
\hline
\end{tabular}

Table 1: Statistics problem instances.

\begin{tabular}{|r|r|r|r|}
\hline Pop Size & Best Value & Worst Value & Mean Value \\
\hline 10 & 3739 & 5506 & 4359.3 \\
\hline 40 & 3404 & 3575 & 3500.1 \\
\hline 70 & 3391 & 3554 & 3449.5 \\
\hline 100 & 3389 & 3407 & 3392.5 \\
\hline 150 & 3389 & 3404 & 3391.9 \\
\hline
\end{tabular}

Table 2: Results CELAR 6 for 10 runs.

Extensive computational experiments have shown that the best solution found by the genetic algorithm is always found within 10 generations for the CELAR instances and for the problem instance GRAPH 5, and within 15 generations for all other GRAPH problem instances.

Let us first demonstrate for problem instance CELAR 6 how the solution quality depends on the population size. For different population sizes we have generated 10 runs, where each run corresponds to applying the genetic algorithm for 10 generations. The best solution value, the worst solution value, and the mean solution value for these 10 runs are given in Table 2 .

It can be seen from Table 2 that the genetic algorithm for CELAR 6 is very stable for population sizes greater than or equal to 100. Tables 3 and 4 show the results of the genetic algorithm with a population size of respectively 100 and 150 for all problem instances. We list the best value, the worst value, the mean value, the number of generations, and the average CPU time per run.

To demonstrate how the solution quality depends on the number of generations we have used the best run with population size 150 for every problem instance. In Table 5 we list the best solution value found after every generation. Note that the best solution value for generation 0 corresponds to the best 1-optimal solution in the initial population.

We can conclude from these computational results that for population sizes of at least 100 the genetic algorithm is very robust, i.e., there is only a small variance in the objective value of the solution produced in each run.

Since no optimal solutions or good lower bounds are known for these problems we can only compare the results with those obtained by other groups in the CALMA project. The genetic algorithm produced the best known solutions to all problem instances. For some problem instances (GRAPH 11 and GRAPH 13) the best objective value found is 


\begin{tabular}{|l|r|r|r|r|r|}
\hline Name & Best Value & Worst Value & Mean Value & Nr Gen & CPU min \\
\hline CELAR 6 & 3389 & 3407 & 3392.5 & 10 & 3 \\
\hline CELAR 7 & 343593 & 353693 & 344784 & 10 & 9 \\
\hline CELAR 8 & 262 & 263 & 262.1 & 10 & 24 \\
\hline GRAPH 5 & 221 & 269 & 240.4 & 10 & 3 \\
\hline GRAPH 6 & 4138 & 5145 & 4431.1 & 15 & 5 \\
\hline GRAPH 11 & 3126 & 4045 & 3468.2 & 15 & 19 \\
\hline GRAPH 13 & 10234 & 11012 & 10486.2 & 15 & 36 \\
\hline
\end{tabular}

Table 3: Results for 10 runs with population size 100.

\begin{tabular}{|l|r|r|r|r|r|}
\hline Name & Best Value & Worst Value & Mean Value & Nr Gen & CPU min \\
\hline CELAR 6 & 3389 & 3404 & 3391.9 & 10 & 5 \\
\hline CELAR 7 & 343592 & 343794 & 343643 & 10 & 13 \\
\hline CELAR 8 & 262 & 263 & 262.2 & 10 & 36 \\
\hline GRAPH 5 & 221 & 236 & 232.5 & 10 & 4 \\
\hline GRAPH 6 & 4125 & 4166 & 4130.7 & 15 & 7 \\
\hline GRAPH 11 & 3088 & 3138 & 3109.5 & 15 & 29 \\
\hline GRAPH 13 & 10110 & 10350 & 10223.1 & 15 & 55 \\
\hline
\end{tabular}

Table 4: Results for 10 runs with population size 150.

\begin{tabular}{|l|r|r|r|r|r|r|r|}
\hline Gen & \multicolumn{1}{|c|}{ C6 } & \multicolumn{1}{|c|}{ C7 } & C8 & GR5 & GR6 & GR11 & GR13 \\
\hline 0 & 8021 & 10272611 & 944 & 6223 & 14814 & 32106 & 50999 \\
\hline 1 & 5686 & 3911098 & 527 & 2927 & 9997 & 25185 & 36809 \\
\hline 2 & 4062 & 748798 & 392 & 1049 & 8104 & 15186 & 31115 \\
\hline 3 & 3710 & 465445 & 299 & 427 & 6667 & 11221 & 24936 \\
\hline 4 & 3430 & 375109 & 278 & 263 & 5699 & 6812 & 20818 \\
\hline 5 & 3423 & 353911 & 270 & 246 & 5251 & 4785 & 15985 \\
\hline 6 & 3392 & 344603 & 266 & 231 & 4879 & 4100 & 13928 \\
\hline 7 & 3391 & 343697 & 264 & 231 & 4434 & 3677 & 11736 \\
\hline 8 & 3389 & 343697 & 262 & 224 & 4187 & 3422 & 10829 \\
\hline 9 & 3389 & 343592 & 262 & 221 & 4132 & 3298 & 10459 \\
\hline 10 & 3389 & 343592 & 262 & 221 & 4130 & 3249 & 10240 \\
\hline 11 & & & & & 4127 & 3208 & 10239 \\
\hline 12 & & & & & 4127 & 3129 & 10152 \\
\hline 13 & & & & & 4127 & 3124 & 10110 \\
\hline 14 & & & & & 4125 & 3223 & 10110 \\
\hline 15 & & & & & 4125 & 3088 & 10110 \\
\hline
\end{tabular}

Table 5: Best solution value per generation. 
$10 \%$ better than the best known solution found by another technique. It still remains a challenge to solve these problem instances to optimality.

\section{References}

[1] K. I. Aardal, C. P. M. van Hoesel, A. M. C. A. Koster, C. Mannino, and A. Sassano. Models and solution techniques for the frequency assignment problem. 4OR, 1(4):261$371,2003$.

[2] K. I. Aardal, C. A. J. Hurkens, J. K. Lenstra, and S. R. Tiourine. Algorithms for radio link frequency assignment: The CALMA project. Operations Research, 50(6):968-980, 2002 .

[3] D. Goldberg. Genetic algorithms in search, optimization $\&$ machine learning. Addison Wesley, 1989.

[4] A. M. C. A. Koster, C. P. M. van Hoesel, and A. W. J. Kolen. The partial constraint satisfaction problem: Facets and lifting theorems. Operations Research Letters, 23(35):89-97, 1998. 